\title{
ALTERNATIVE WAYS OF ESTIMATING THE NUMBER OF GENES IN A POLYGENIC SYSTEM BY GENOTYPE ASSAY
}

\author{
PHILOMENA TOWEY and J. L. JINKS \\ Department of Genetics, University of Birmingham, Birmingham BI5 2TT, England
}

Received 1.iv.77

\begin{abstract}
Summary
The procedures for estimating $k$, the number of genes, or more strictly the number of effective factors in a polygenic system by the method of genotype assay have been extended to any number, $p$, of $\mathrm{F}_{n+2}$ grand progeny families raised from each $F_{n}$ individual assayed. Formulae are also derived that would be more appropriate for estimating $k$ if dominance were absent or in the more unlikely event of no internal balancing.

The existing and new procedures are illustrated by the analysis of data from a cross between varieties 1 and 5 of Nicotiana rustica which extended to the $\mathrm{F}_{8}$ generation. The structure of these data permitted the estimation of $k$ for flowering time and final height in the $\mathrm{F}_{2}$ from assessments made on the $\mathrm{F}_{4}$ for $p=2$, from $\mathrm{F}_{5}$ to $\mathrm{F}_{8}$ for $p=4$ and from joint consideration of the $\mathrm{F}_{4}$ to $\mathrm{F}_{8}$ assessments. All these assays of the $\mathrm{F}_{2}$ gave low estimates for $k$. On the other hand, using the same $F_{5}$ to $F_{8}$ data to assay the $F_{3}$ to $F_{6}$ generations respectively for $p=2$ gave estimates of $k$ that increased rapidly with generation so that for every one detected in the $F_{2}$ there were ten on average in the $F_{6}$.

Checks and controls of the material and method, including using the same procedures to estimate $k$ for a known single major gene difference segregating in this cross, leave no doubt that the rise in the estimate is genuine. Furthermore, it is expected from the nature of effective factors and the linkage disequilibrium that is generated on making this cross and subsequently resolved over successive rounds of recombination.

These analyses confirm the overwhelming superiority of genotype assay over the other methods of estimating $k$ that are available in all but a few species.
\end{abstract}

\section{INTRODUGTION}

Jinks AND TOWEy (1976) described a new approach to estimating $k$, the number of genes, or more correctly effective factors in a polygenic system using genotype assay. Basically, each individual from a random sample of $m$ individuals of the $\mathbf{F}_{n}$ generation is assayed for evidence of heterozygosity through two of its randomly chosen grand-progeny families of the $F_{n+2}$ generation. This, however, is but one special case of a general procedure in which the $m \mathrm{~F}_{n}$ individuals are each assayed through $p$ grand-progeny families each consisting of $l$ sibs. Within the same total number of $m p l$ individuals in the $F_{n+2}$ generation there are many ways of deploying resources that will have consequences for the reliability of the estimate of $k$. In this paper we consider some of the theoretical consequences of varying $p$ while keeping $m$ and $l$ constant and also the genetical situations in which intermediate probabilities are more appropriate than $\mathrm{P}_{\mathrm{Max}}$ for estimating $k$.

The procedures are illustrated by the analysis of Nicotiana rustica breeding programmes based upon the cross $\mathrm{Vl} \times \mathrm{V} 5$ (Mather and Vines, 1952). This analysis provides overwhelming confirmation that the number of 
effective factors increases over the successive generations derivable from an initial cross.

\section{Theory}

\section{(i) Setting the limits}

Jinks and Towey (1976) gave the probability, $\mathrm{P}_{\text {Het. } r}$, that a heterozygote in the $n$th generation would be heterozygous at $r$ of the $k$ loci, where $r$ could take all values from $I$ to $k$ as

$$
\mathrm{P}_{\text {Het. } r}=\frac{1}{(2 n-1)} k^{k} C_{p}\left(2^{n-1}-1\right)^{k-r} .
$$

This expression depends on $n$, the generation, and $k$ only. However, the probability of detecting differences between the $p$ individuals chosen at random from the $\mathrm{F}_{n+1}$ progeny of a selfed heterozygote in the $\mathrm{F}_{n}$ generation is dependent on $p$ and in our earlier paper we considered only the spccial case of $p=2$. Where each genotype has a unique phenotype the probability of detecting differences among $p$ individuals will be related to $r$, the number of loci at which the grand-parent was heterozygous, as

$$
1-\left(\frac{2^{p}+2}{4^{p}}\right)^{r}
$$

From which it follows that the frequency of heterozygotes $\left(\mathrm{P}_{\mathrm{Max}}\right)$ in the $\mathrm{F}_{n}$ generation that is detectable by progeny testing $p \mathrm{~F}_{n+2}$ random progenies of each individual in the $\mathrm{F}_{n}$ generation will be

$$
\mathrm{P}_{\mathrm{Max}}=\frac{1}{\left(2^{n-1}\right)^{k}} \sum_{r=0}^{k}{ }^{k} C_{r}\left(2^{n-1}-1\right)^{k-r}\left(1-\frac{2^{p}+2}{4^{p}}\right)^{r},
$$

which simplifies to

$$
\mathrm{P}_{\mathrm{Max}}=1-\left(1+\frac{2^{p}+2-4^{p}}{2^{n+2 p-1}}\right)^{k}
$$

If, following Jinks and Towey, the effects of both internal and rclational balance are taken into account the probability that $p$ individuals chosen at random from the $F_{n+1}$ progeny of a selfed heterozygote will give progenies that differ becomes

$$
1-\frac{1}{4^{p k}} \sum_{r=0}^{k}\left(\frac{3^{r} k !}{r !(k-r) !}\right)^{p}
$$

and the frequency of heterozygosity in the $n$th generation that will be detectable by our proccdures is then,

$$
\mathrm{P}_{\mathrm{Min}}=1-\left(1-\frac{1}{2^{n-1}}\right)^{k} \sum_{r=0}^{k} \frac{{ }^{k} C_{r} \sum_{s=0}^{r} 3^{p s}\left({ }^{r} C_{s}\right)^{p}}{\left[2^{n+2 p-1}-2^{2 p}\right]^{r}} .
$$

In fig. 1 are plotted the valucs of $\mathrm{P}_{\mathrm{Max}}$ and $\mathrm{P}_{\mathrm{Min}}$ for $k=1$ to $20, n=2$ and 5 and $p=2$ and 4 . For lower values of $k$ both $\mathrm{P}_{\operatorname{Max}}$ and $\mathrm{P}_{\mathrm{Min}}$ arc more scnsitive to changcs in $k$ for $p=4$ than for $p=2$. Futhermore, the differences bctween the $\mathrm{P}_{\mathrm{Max}}$ and $\mathrm{P}_{\mathrm{Min}}$ curves are less for $p=4$ than for 


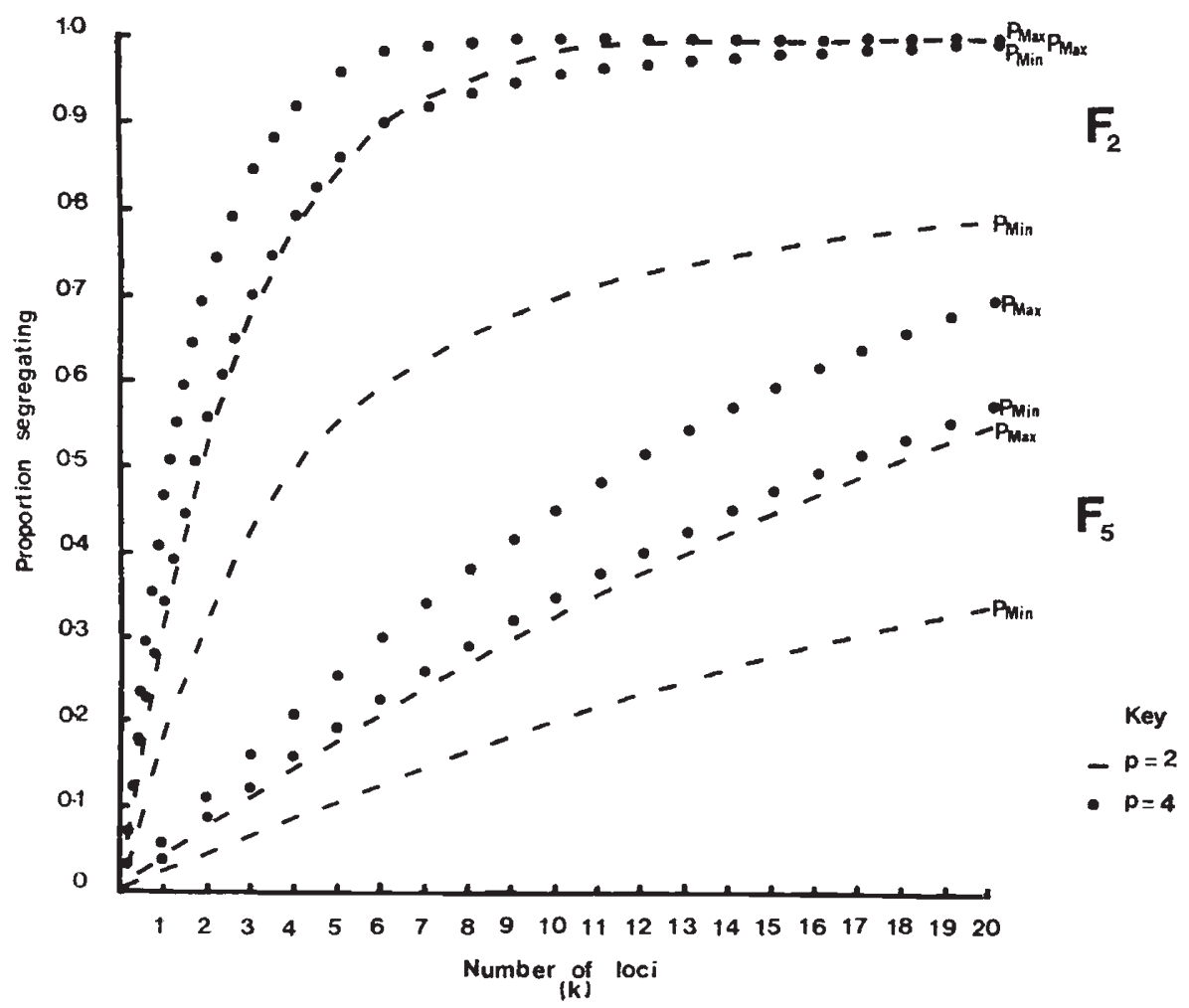

Frg. 1.- The effect of varying $p$, the number of $\mathrm{F}_{n+1}$ individuals chosen from a selfed individual in generation $n$, on the relationship between the proportion of detectable segregations and $k$, the number of loci for the maximum and minimum curves. Two values of $p$ and $n$ are used to illustrate the effect.

$p=2$, and hence the estimates are less sensitive to the assumptions which distinguish them.

\section{(ii) Intermediate situations}

$P_{M i n}$, which sets the upper limit to the number of effective factors, is based upon the supposition that both internal balance and dominance are operating simultaneously to minimise the number of different genotypes which have different phenotypes. Where there is prior knowledge of the dominance relationships, alternative formulae may be more appropriate. And while it is very unlikely that prior knowledge of the extent of internal balancing would be available it is illuminating to consider the consequences of modifying this assumption.

If there is little or no dominance, so that the phenotype of the heterozygote for any locus is distinct from that of either homozygote but there is maximum internal balance, i.e. $d_{a}=d_{b}=d_{c} \ldots=d_{k}$ the $\mathrm{P}_{\text {Min }}$ becomes

$$
\mathbf{P}_{\text {Int A }}=1-\left(1-\frac{1}{2^{n-1}}\right)^{k} \sum_{r=0}^{k} \frac{{ }^{k} C_{r} \sum_{s=0}^{2 r}\left({ }^{2 r} C_{s}\right)^{p}}{\left[2^{n+2 p-1}-2^{2 p}\right]^{r}} .
$$


The alternative situation, namely, high dominance $(h=2 d$, not $h=d$ as stated by Jinks and Towey, 1976) but no internal balance can also be specified by modifying the PMin to become

$$
P_{\text {Int. B }}=1-\left(1+\frac{3^{p}+1-4^{p}}{2^{n+2 p-1}}\right)^{k} \text {. }
$$

But it is doubtful whether in practice there could ever be a situation in which there is no internal balance unless $k$ is very small. For example, for $k=2$ the condition for no internal balance is simply $d_{a} \neq d_{b}$ while for $k=3$ the conditions required are $d_{a} \neq d_{b} \neq d_{c}, d_{a} \neq d_{b}+d_{c}, \pm d_{a} \neq d_{b}-d_{c}$, $d_{b} \neq d_{a}+d_{c}, \pm d_{b} \neq d_{a}-d_{c}, d_{c} \neq d_{a}+d_{b}$ and $\pm d_{c} \neq d_{a}-d_{c}$. For $k=4$ the number of conditions grows correspondingly since they include in addition to $d_{a} \neq d_{b} \neq d_{c} \neq d_{d}$ all possible combinations of the types $d_{a} \neq d_{b}+d_{c}+d_{d}$, $d_{a} \neq d_{b}-d_{c}+d_{d}$ and $d_{a} \neq d_{b}-d_{c}-d_{d} ; d_{a}+d_{b} \neq d_{c}+d_{d}$ and $d_{a}+d_{b} \neq d_{c}-d_{d}$. Clearly, as $k$ increases the number of conditions that have to be simultaneously met to ensure no internal balancing becomes impossibly large. In practice, therefore, some internal balancing must always be occurring even when $d_{a} \neq d_{b} \neq d_{c} \ldots \neq d_{k}$ unless $k$ is very small. The true upper limit to the estimate of $k$ must therefore be somewhere between that obtained from $P_{\text {Min }}$ and $P_{\text {Int. }} \mathrm{B}$ but probably closer to that from $\mathrm{P}_{\mathrm{Min}}$.

The use of $P_{\text {Min }}$ or $P_{\text {Int. B }}$ to set the upper limit to the value of $k$ strictly applies only if the detection of heterozygotes in the $\mathrm{F}_{n}$ depends solely on the finding of differences among the means of the $p \mathrm{~F}_{n+2}$ families derived from each $\mathrm{F}_{n}$ grandparent. They are not the appropriate limits, however, if we also detect heterozygotes by finding differences among the variances within the $p \mathrm{~F}_{n+2}$ families. Thus while dominance reduces the probability of observing differences between a homozygous dominant and a heterozygote on the basis of their phenotypic contributions to family means there is no corresponding reduction arising from their contribution to the within family variances. We should not use variances therefore, in conjunction with the $\mathrm{P}_{\mathrm{Min}}$ or $\mathrm{P}_{\text {Int. } \mathrm{B}}$ formulae. In practice the issue does not arise because we rarely if ever detect a difference between family variances without also detecting a difference in the family means because of the greater sensitivity of the latter. We frequently, however, detect differences between family means without finding a difference between the corresponding family variances (see Jinks and Towey, 1976, Table 2).

In fig. 2 we present $\mathrm{P}_{\text {Int. } \mathrm{A}}$ and $\mathrm{P}_{\text {Int. } \mathrm{B}}$ for $n=2$ and $5, k=1$ to 20 and $p=2$ along with $\mathrm{P}_{\mathrm{Max}}$ and $\mathrm{P}_{\mathrm{Min}}$ for comparison. Although $\mathrm{P}_{\text {Int. A }}$ and $P_{\text {Int. }} \mathrm{B}$ give probabilities which fall between those of $\mathrm{P}_{\mathrm{Max}}$ and $\mathrm{P}_{\mathrm{Min}}$, $P_{\text {Int. } \mathrm{B}}$ is almost the same as $\mathrm{P}_{\text {Min }}$ and deviates from it only as $n$ decreases

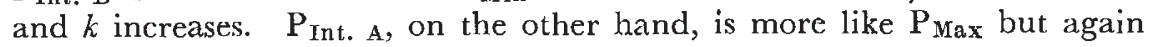
it falls progressively below this value as $n$ decreases and $k$ increases.

\section{Materiats and method}

Appropriate material for illustrating the usc of the probability curves for estimating $k$ and the effect of varying $p$ against a constant total of $p m l$ individual plants in the generation of assessment is provided by the cross between V1 and V5 of Nicotiana rustica initiatcd in 1944 by Professor K. Mather and his colleagues to study the variation in flowering time and 
final height (see Mather and Vines, 1952; Breese, 1954). The structure of the experiment which continued to the $F_{8}$ generation grown in 1952 is shown in fig. 3 taken from Breese (1954). Beyond the $\mathrm{F}_{2}$ only one of the 20 groups of families is illustrated, a group being all the descendants of one of the $20 \mathrm{~F}_{2}$ individuals. Each group is divisible into two sub-groups, the members of each being the descendants of a single $F_{3}$ individual. From the $F_{5}$ onwards the experiment was designed to have 20 such groups each consisting of two sub-groups and each sub-group consisting of two families, although it fell below this in the later generations because of random losses

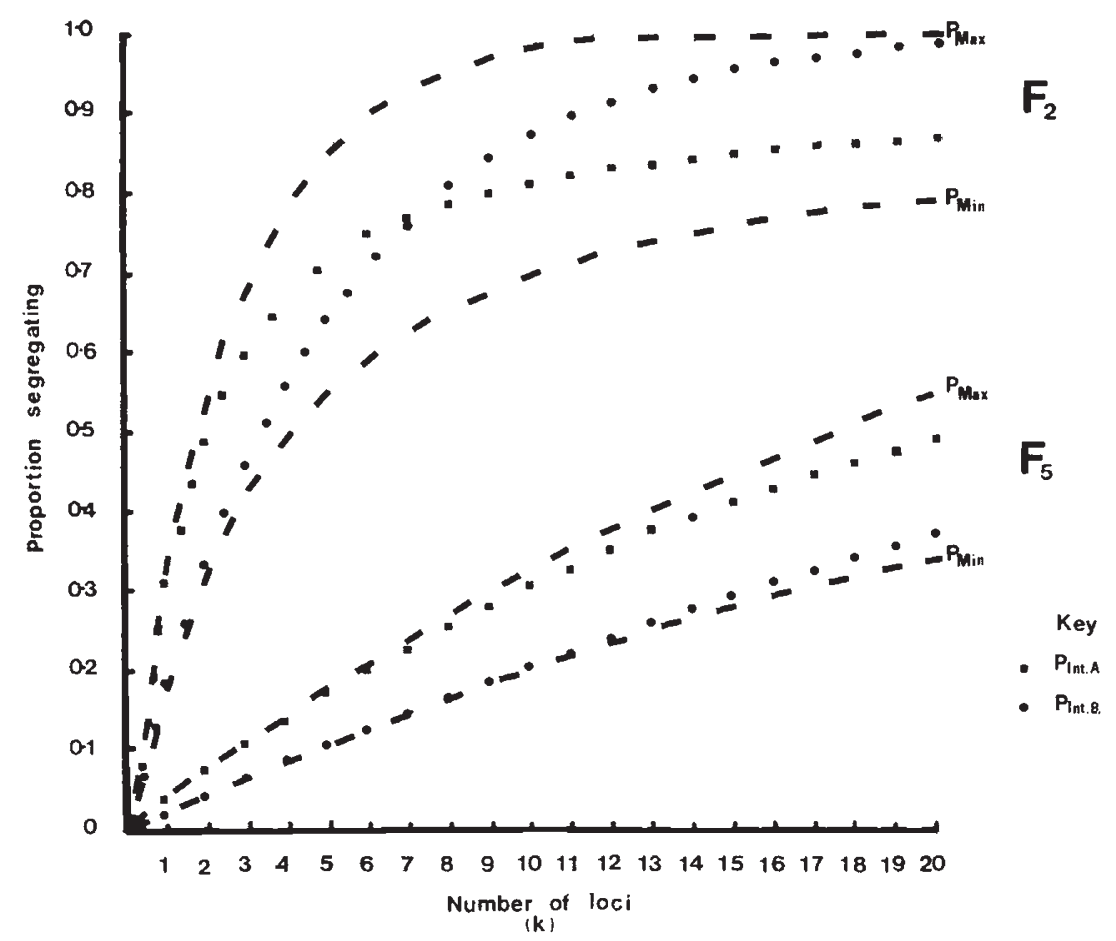

Fig. 2.-The relationship between the proportion of detectable segregations and $k$, the number of loci for the maximum and minimum curves, and for the two intermediates for $p=2$. The $\mathrm{F}_{2}$ and $\mathrm{F}_{5}$ generations are again used for illustration (see fig. 1).

(see table 1). The unit of randomisation throughout was a plot of five plants of the same family and one plot per family was raised in each of the two independently randomised blocks.

The generations up to the $F_{4}$ were grown at Merton, London, and the $F_{5}$ to $F_{8}$ at Winterbourne, Birmingham. Because of practical difficulties which arose during the transition, the $F_{5}$ generation which provided the parents of the $F_{6}$ were raised in 1949 (see fig. 3) but the $F_{5}$ data we shall place most reliance upon for assaying the heterozygosity in the $F_{3}$ were a replicate sample of the $F_{5}$ families grown along with the $F_{6}$ in 1950 .

We can estimate $k$ for the $\mathrm{F}_{2}$ to $\mathrm{F}_{6}$ generations by analysing their $\mathrm{F}_{4}$ to $F_{8}$ grand-progeny families for $p=2$. Since, however, from the $F_{5}$ to $F_{8}$ generations each $\mathrm{F}_{2}$ individual is represented by a group of four families 
we can use the $F_{5}$ to $F_{8}$ generations with $p=4$ to detect segregation and hence estimate $k$ for the $\mathrm{F}_{2}$, that is, use the $\mathrm{F}_{n+2}$ to $\mathrm{F}_{n+5}$ generations to cstimate $k$ for constant $\mathbf{F}_{n}=\mathbf{F}_{2}$ and constant $p m l$ apart from random losses for $p=4$. Hence the only variable that could affect the estimates of $k$

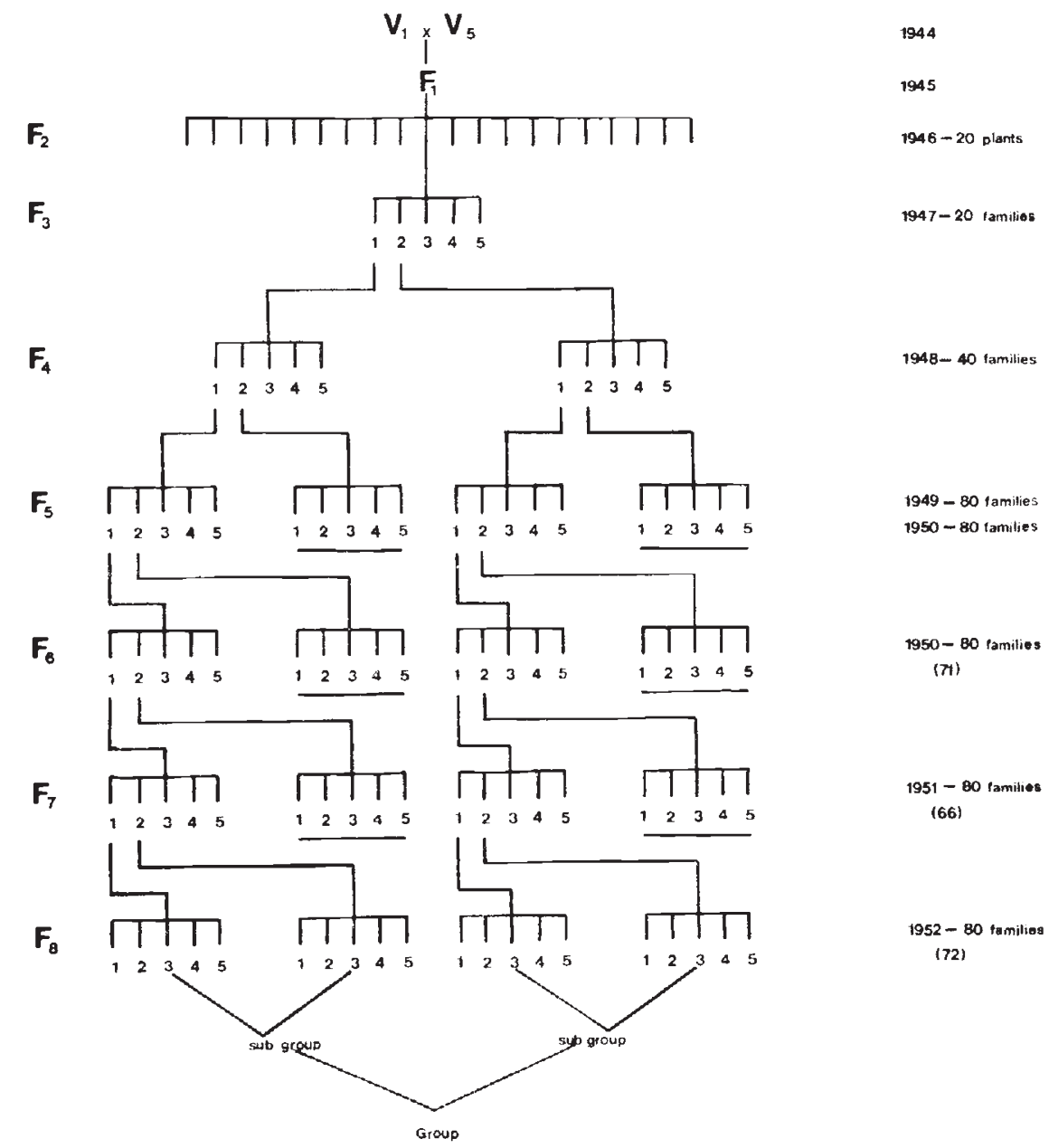

FIG. 3.-Genealogical table showing how a single group of families is derived from an $F_{2}$ individual. Each group consists of two sub-groups each derived from an $\mathrm{F}_{3}$ individual. Twenty such groups each consisting of two sub-groups wcre initiated in the early generations. Each family is represented by a plot of five plants. The potential numbcr of families are given for each gencration together with the actual number achieved (Breese, 1954).

would be any changes in the sensitivity with which we can detect differences between family means from the $F_{5}$ to the $F_{8}$ generations. We would, for example, expect an increasc in sensitivity because of the expected decrease in the variances of the family means as the families become more inbred. But superimposed upon this there is the unpredictable effects of seasonal 
differences over the three years, 1950, 1951 and 1952, in which they were grown.

For the estimates based upon $p=2$, each of the pairs of families within a sub-group was subjected to an analysis of variance in which four items were recognised; the difference between the means of the two families for 1 degree of freedom, the difference between the two blocks for 1 degree of freedom, the interaction between families and blocks for 1 degree of freedom and the differences between individuals within families within blocks ( = within plots) for 16 degrees of freedom. If the block interaction mean square was not significant when tested against the within family within

TABLE 1

Summary of the stages in the estimation of $\mathrm{k}$, the number of effective factors, for flowering time and final height in the $F_{2}$ to $F_{6}$ generations for $\mathrm{p}=2$. Estimates for $\mathrm{k}$ in the $F_{3}$ based upon the $F_{5}$ raised in 1949 are given in brackets

Character

$\mathbf{F}_{n}$ generation: $m$ in $\mathbf{F}_{n+2}$ generation:

$\mathrm{P}$ for $\leqq 0.05$

for $\leqq 0 \cdot 01$

$$
\begin{array}{r}
k \text { from } P_{M a x} \\
\text { for } \leqq 0.05 \\
\text { for } \leqq 0.01 \\
k \text { from } P_{M i n} \\
\text { for } \leqq 0.05 \\
\text { for } \leqq 0.01
\end{array}
$$

for $\mathbf{F}_{n+2}$ generation

$$
\begin{aligned}
& E_{2} \\
& \sigma_{\omega}^{2}
\end{aligned}
$$

Smallest significant difference
Flowering time

$\begin{array}{ccccc}\mathbf{F}_{2} & \mathbf{F}_{3} & \mathbf{F}_{4} & \mathbf{F}_{5} & \mathbf{F}_{6} \\ 20 & 40 & 35 & 30 & 36 \\ 0.200 & 0.450 & 0.314 & 0.233 & 0.306 \\ 5 & (0.200) & & \\ 0.200 & 0.250 & 0.086 & 0.166 & 0.166 \\ & (0.125)\end{array}$

$\begin{array}{rrrrr}1 & 4(2) & 5 & 7 & 19 \\ 1 & 2(1) & 2 & 5 & 10 \\ & & & & \\ 2 & 8(3) & 9 & 12 & >20 \\ 2 & 4(2) & 2 & 8 & 16 \\ & & & & \\ 2.58 & 4.55 & 4.55 & 7 \cdot 87 & 3.31 \\ 7.52 & 22.39 & 14.86 & 9 \cdot 62 & 9.92 \\ & & & & \\ 3.30 & 2 \cdot 40 & 2.50 & 2.50 & 2 \cdot 00\end{array}$

Final height

$\begin{array}{lllll}\mathbf{F}_{2} & \mathbf{F}_{3} & \mathbf{F}_{4} & \mathbf{F}_{5} & \mathbf{F}_{6} \\ 20 & 40 & 35 & 30 & 36 \\ 0.400 & 0.300 & 0.257 & 0.200 & 0.306 \\ 5 & (0.200) \\ 0.250 & 0 \cdot 17.5 & 0.174 & 0.166 & 0.250 \\ & (0 \cdot 125) & & & \end{array}$

$\begin{array}{rrrrr}2 & 3(2) & 4 & 6 & 19 \\ 1 & 2(1) & 3 & 5 & 15 \\ & & & & \\ 3 & 4(3) & 7 & 10 & >20 \\ 2 & 2(2) & 5 & 8 & >20\end{array}$

$3 \cdot 83$

$\begin{array}{llll}13 \cdot 12 & 13 \cdot 12 & 7 \cdot 00 & 4 \cdot 29\end{array}$

$\begin{array}{llll}27 \cdot 27 & 20.95 & 12 \cdot 10 & 10 \cdot 56\end{array}$

$\begin{array}{lllll}4 \cdot 10 & 3.98 & 4 \cdot 60 & 3.80 & 2 \cdot 58\end{array}$

blocks mean square the latter was used to test the difference between the two families. If, however, the block interaction was itself significant it was used to test the difference between the two families, leading to a variance ratio for one and one degree of freedom. This made it practically impossible to find a difference between the families whenever the block interaction was significant.

For the estimates based upon $p=4$ each set of four families within a group was subjected to an analysis of variance in which the same four items were recognised and the same comparisons were made but with $3,1,3$ and 32 degrees of freedom, respectively.

In addition to flowering time and final height, one further character was recorded by Mather and Vines and Breese, namely, the presence or absence of anthocyanin on the ovary. This is controlled by a single major gene difference $A$ - $a$, with the presence of anthocyanin dominant to its absence (Mather and Vines, 1952). We can, therefore, use this character as a 
control for testing both the material and the method. We can use its segregation in the $\mathrm{F}_{n+2}$ to detect heterozygosity in the $\mathrm{F}_{n}$ generation and to estimate the proportion of heterozygotes for both $p=2$ and $p=4$. Furthermore, we can base our estimate solely on phenotype differences, i.e. wherc we do not distinguish $A A$ and $A a$, in which case the $\mathrm{P}_{\mathrm{Min}}$ formula for estimating $k$ is appropriate or alternatively use evidence of subsequent segregation to distinguish $A a$ from $A A$, in which casc the appropriate formula for estimating $k$ is $\mathrm{P}_{\text {Max }}$.

\section{Estimates}

In table 1 are presented the critical steps in the estimation of $k$, the number of effective factors, for flowering time and final height in the $\mathrm{F}_{2}$ to $F_{6}$ generations, based upon comparisons of thcir $F_{4}$ to $F_{8}$ grand-progenies, respcctively, for $p=2$. For example, in the $F_{5}$ grown in 1950, 40 pairs of families, each pair having the same $\mathrm{F}_{3}$ grandparent, were raised. For flowering time 18 of these 40 pairs had significantly different means with a probability of 5 per cent or less $(\leqq 0.05)$, and ten at a probability of 1 per cent or less $(\leqq 0 \cdot 001)$. Thus the proportion $P$ of $\mathrm{F}_{3}$ plants which were demonstrably heterozygous was 0.450 at the 5 per cent level of significance and 0.250 at the 1 per cent level. If we assume that all genotypic differences lead to phenotypic differences, these proportions are estimates of $\mathrm{P}_{\mathrm{Max}}$ which in turn takes the values expected for $k=4$ and 2, respectively. If, on the other hand, we assume that a proportion of the genotypic differences are not displayed as phenotypic differences consistent with complete dominance and equal additive genetic effects at all loci, these proportions are estimatcs of $\mathrm{P}_{\mathrm{Min}}$ and these estimates are as expected for $k=8$ and 4 respectively. The other estimates in table 1 have been similarly computed. Since in the seasons in which the assessments were made the $\mathrm{V} 1 \times \mathrm{V} 5$ cross displayed dominance for early flowering and for greater final height (Breese, 1954; Bucio Alanis, Perkins and Jinks, 1969), PMin would seem to be more appropriate for estimating $k$ than either $\mathrm{P}_{\mathrm{Max}}$ or $\mathrm{P}_{\text {Int }}$.

In table 2 are presented the corresponding stages in the estimation of $k$ for flowering time and final height in the $F_{2}$ based upon assessments of $F_{5}$ to $F_{8}$ progenies for $p=4$. Comparisons of the estimates in tables 1 and 2 present some remarkablc features. The estimates of $k$ in the $\mathrm{F}_{2}$ are low whether estimated from the $\mathrm{F}_{4}$ for $p=2$ (table 1 ) or from the $\mathrm{F}_{5}$ to $\mathrm{F}_{8}$ for $p=4$ (tablc 2). In complete contrast, the estimates of $k$ in the $F_{3}$ to $F_{6}$ generations based on $F_{5}$ to $F_{8}$, rcspectively for $p=2$, show a marked and progressive increase with generation (table 1) even though they are based on the same observations as the $\mathrm{F}_{2}$ estimates in table 2. There are two possible causes of any change in $k$ with generation, one of which is common to both sets of estimates and the other unique to the set in table 1 . The common causc is changes in the sensitivity with which differences between family means can be detected in the $\mathrm{F}_{n+2}$ generation of assessment, i.e. $\mathrm{F}_{4}$ to $\mathrm{F}_{8}$. Statistics relating to this cause are summarised in the bottom three rows of table 1 but thcy also relate to the corresponding $F_{n+2}$ columns of table 2.

The first of these statistics, $E_{2}$, measures the variation among family means that is expected to arise solely from non-heritable causes (Mather and Jinks, 1971). Any difference between family means must be significantly 
greater than this before we can infer heritable differences. The estimates of $E_{2}$ which are taken from Mather and Vines (1952) and Breese (1954) vary markedly between the $\mathrm{F}_{4}$ and $\mathrm{F}_{8}$ generations, but major trends are discernible. For flowering time there is an increase from the $F_{4}$ to $F_{7}$ followed by a sharp drop to the $F_{8}$. These changes are presumably related to seasonal differences. For final height there is an abrupt increase between the $\mathrm{F}_{4}$ and the $\mathrm{F}_{5}$ coinciding with the move from Merton to Winterbourne (see Section 3 ), followed by a steady decline as the new site was developed. These changes presumably reflect the level of homogeneity of the soil conditions.

The second of these statistics $\sigma_{\omega}^{2}$ measures the average within family variation arising from all causes both heritable and non-heritable in the $\mathrm{F}_{n+2}$ generation. It is a component of the sampling error of the family means and hence contributes to the sensitivity with which we can detect

TABLe 2

Summary of the stages in the estimation of $\mathrm{k}$, the number of effective factors for flowering time and final height in the $F_{2}$ using the $F_{5}$ to $F_{8}$ generations for $\mathrm{p}=4$. Estimates based on the $F_{5}$ raised in 1949 are given in brackets

\begin{tabular}{|c|c|c|c|c|c|c|c|c|}
\hline Character & \multicolumn{4}{|c|}{ Flowering time } & \multicolumn{4}{|c|}{ Final height } \\
\hline Generation of assessment: & $\mathrm{F}_{5}$ & $F_{6}$ & $\mathrm{~F}_{7}$ & $F_{8}$ & $\mathrm{~F}_{5}$ & $\mathrm{~F}_{6}$ & $\mathrm{~F}_{7}$ & $\mathrm{~F}_{8}$ \\
\hline$m:$ & 20 & 15 & 13 & 16 & 20 & 15 & 13 & 16 \\
\hline$P$ for $\leqq 0.05$ & $\begin{array}{c}0.850 \\
(0.500)\end{array}$ & 0.866 & 0.769 & 0.875 & $\begin{array}{c}0.550 \\
(0.600)\end{array}$ & 0.666 & 0.615 & 0.563 \\
\hline for $\leqq 0.01$ & $\begin{array}{c}0 \cdot 700 \\
(0 \cdot 100)\end{array}$ & 0.400 & 0.769 & 0.313 & $\begin{array}{c}0.500 \\
(0.300)\end{array}$ & $0 \cdot 266$ & 0.462 & 0.563 \\
\hline $\begin{array}{r}k \text { from } P_{\operatorname{Max}} \\
\text { for } \leqq 0.05 \\
\text { for } \leqq 0.01\end{array}$ & $\begin{array}{l}4(2) \\
2(1)\end{array}$ & $\begin{array}{l}4 \\
1\end{array}$ & $\begin{array}{l}3 \\
3\end{array}$ & $\begin{array}{l}4 \\
1\end{array}$ & $\begin{array}{l}2(2) \\
2(1)\end{array}$ & $\begin{array}{l}2 \\
1\end{array}$ & $\begin{array}{l}2 \\
1\end{array}$ & 2 \\
\hline $\begin{array}{r}k \text { from } P_{\text {Min }} \\
\text { for } \leqq 0.05 \\
\text { for } \leqq 0.01\end{array}$ & $\begin{array}{l}5(2) \\
3(1)\end{array}$ & $\begin{array}{l}6 \\
2\end{array}$ & $\begin{array}{l}4 \\
4\end{array}$ & $\begin{array}{l}6 \\
1\end{array}$ & $\begin{array}{l}2(3) \\
2(1)\end{array}$ & $\begin{array}{l}3 \\
1\end{array}$ & $\begin{array}{l}3 \\
2\end{array}$ & \\
\hline
\end{tabular}

differences between them. Indeed, in the absence of block interactions it is the sole component of the error variance used to detect segregation.

The estimates in table 1 are again taken from Mather and Vines (1952) and Breese (1954). For both characters they show an abrupt increase between the $F_{4}$ and $F_{5}$ followed by a gradual decrease. The heritable component of this variance, of course, halves with every generation of selfing and this contributes to the steady fall from the $F_{5}$ to $F_{8}$. The large increase between $F_{4}$ and $F_{5}$ presumably results from the change in location noted earlier.

The cumulative effects of these changes is revealed by the third statistic in table 1 , which is the smallest difference between any pair of family means which proved to be significant at the 5 per cent level $(\leqq 0 \cdot 05)$. For flowering time this decreases from the $F_{4}$ to the $F_{8}$ but for final height it reaches its greatest value in the $\mathrm{F}_{6}$ before falling to a relatively low value by the $\mathrm{F}_{8}$.

If we examine the estimates of $k$ in table 2, for which $\mathrm{F}_{n}$ is constant but the generation of assessment is changing, we find no trends in the estimates for either character at the 1 per cent or the 5 per cent levels of 
significance. Indeed, at the latter level the estimates are remarkably stable over the four generations used for assessment. There is no evidence, therefore, that changes in sensitivity are markedly affecting these estimates of $k$.

Since the samc $F_{5}$ to $F_{8}$ data are also used to obtain the estimates of $k$ in table 1 we do not expect any marked trends in these estimates arising from changes in sensitivity. The large and consistent increases in the estimates with gcneration shown by both characters at both levels of significance must therefore arise from other causes. Since the estimates in table 2 differ from those in table 1 solely in that $F_{n}$ is increasing from $F_{2}$ to $F_{6}$, the cause of this rapid upward trend in the estimates can only be due to this. For different $\mathrm{F}_{n}$ 's we use different curves relating $\mathrm{P}_{\mathbf{M a x}}$ and $\mathrm{P}_{\mathrm{Min}}$ to $k$ and the relative accuracies of the estimates in different parts of the range of $k$ values varies with $n$ (Jinks and Towey, 1976; and figs. 1 and 2). This could not, however, account for the ten-fold or greater increases in estimates from the $\mathrm{F}_{2}$ to the $\mathrm{F}_{6}$. Most of this increase must, therefore, bc genuine and result from the successive rounds of recombination that occur during the production of the $\mathrm{F}_{2}, \mathrm{~F}_{3}, \mathrm{~F}_{4}$ and $\mathrm{F}_{5}$ gametes.

As a final check on the material and method we have estimates $k$ for the single major gene controlled presence and absence of anthocyanin (Section 3). For example, if we use the 20 pairs of $F_{4}$ families $(p=2)$ to determine the detectable heterozygosity for this character in the $\mathrm{F}_{2}$ on the basis of phenotypic differences only, the proportion of heterozygotes is $0 \cdot 10$ ( 2 out of 20). Equating this to $P_{\text {Min }}$ gives an estimate of $k=1$. If on the basis of subsequent segregation we distinguish heterozygotes from the dominant homozygote the proportion of detectable heterozygosity in the $\mathrm{F}_{2}$ rises to 0.40 ( 8 out of 20). This is now an estimate of $P_{M a x}$ and as such it gives an estimate of $k=2$, the actual value falling between 1 and 2 . If for example we now use the alternative estimate of $k$ in the $\mathrm{F}_{2}$ based upon $p=4$ in the $\mathrm{F}_{8}$ and again confine ourselves to phenotypic differences the proportion of detectable heterozygotes is 0.25 , which being an estimate of $\mathrm{P}_{\text {Min }}$ gives $k=1$. If, on the other hand, we now use the same $\mathbf{F}_{8}$ data to estimate $k$ in the $\mathrm{F}_{6}$ we obtain an estimate of $k=0$ for $\mathrm{P}_{\mathrm{Min}}$ although the subsequent segregation of a hcterozygote would give a $\mathrm{P}_{\operatorname{Max}}$ estimate of $k=1$. These estimates give no reason for doubting either the rcliability of the material or the validity of this method of analysis.

\section{Pedigree analysis}

The pedigree of every $F_{3}$ to $F_{8}$ family can be traced back to onc of $20 F_{2}$ individuals (fig. 3), hence we can combine the assessments already made on the $\mathrm{F}_{4}$ to $\mathrm{F}_{8}$ generations to individually assay cach of the $\mathrm{F}_{2}$ individuals for hetcrozygosity. For each $\mathrm{F}_{2}$ we have five separate occasions from the $\mathrm{F}_{4}$ to $\mathrm{F}_{8}$ to detcrmine whether progenies derived from it are segregating, and evidence of segregation at any one of these fivc occasions is sufficient to establish that the $\mathrm{F}_{2}$ plant was a hetcrozygote.

In table 3 arc given the distributions among the $20 \mathrm{~F}_{2}$ groups of the number of occasions for which there is evidence of segregation at the 5 and 1 per ccnt levcls of significance. The proportion showing one or more significances can bc regarded as estimates of $\mathrm{P}_{\operatorname{Max}}$ or $\mathrm{P}_{\mathrm{Min}}$ for $p=4$ sincc therc are four families per generation for all but the $F_{4}$ for each $F_{2}$ group. These estimates equate to the values of $k$ given in table 3 . For the 5 per cent signi- 
TABLE 3

The distributions of the number of occasions over the $F_{4}$ to $F_{8}$ generations of the 20 pedigrees (fig. 3) for which there is evidence of segregation at the 5 and 1 per cent levels of significance for flowering time and final height and estimates of $\mathrm{k}$ based upon them for $P_{\mathrm{Max}}$ and $P_{\mathrm{Min}}$

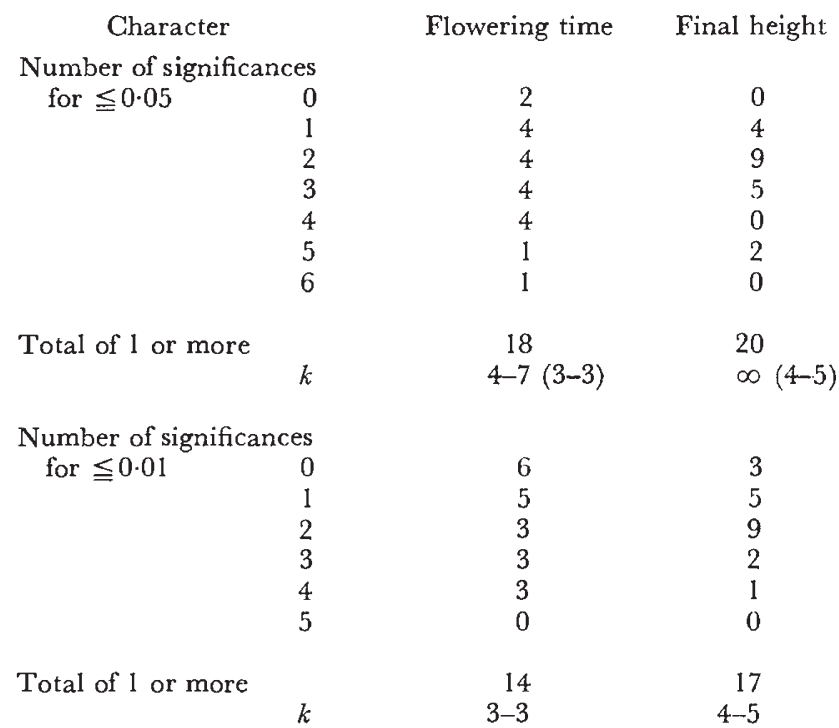

ficance level two pairs of estimates are given, the second pair in brackets being those obtained if we omit $\mathrm{F}_{2}$ 's for which the evidence for segregation is a single 5 per cent significance. The only effect of any consequence of doing this is to bring the indeterminate estimate for final height into line with the other estimates.

\section{Conclusions}

The most significant finding of this study is the unambiguous demonstration that the number of effective factors increases steadily over successive generations of selfing following an initial cross. Although we reached this same conclusion from our earlier study of a completely independent cross (Jinks and Towey, 1976) we did not then have the range of internal checks and controls of both the material and the method that the present data provide. Thus we have been able to demonstrate the soundness of the data and our procedures by using a known single major gene difference controlling anthocyanin production. We have also been able to demonstrate the relatively low estimate of the number of effective factors in the $F_{2}$ by three different procedures which use all the data available in one form or another. In so doing we have shown that the low estimate in the $\mathrm{F}_{2}$ is quite unrelated to the generation used to assess the $F_{2}$. Since all the data used to estimate the number of effective factors in the later generations were also used to estimate the number in the $F_{2}$ there can be no explanation of the higher estimates in the $\mathrm{F}_{3}, \mathrm{~F}_{4}, \mathrm{~F}_{5}$ and $\mathrm{F}_{6}$ other than that they are genuinely higher.

The explanation of this increase in the number with generation rests upon the nature of effective factors (Mather and Jinks, 1971) and the fact

$39 / 3-\mathrm{G}$ 
that strong linkage disequilibrium can be initially generated in a cross between a pair of lines and subsequently resolved during successive rounds of recombination. That there is linkage disequilibrium for the genes controlling the variation in $\mathrm{FT}$ and $\mathrm{FH}$ in this cross has been demonstrated on a number of occasions (scc Perkins and Jinks, 1970). In these circumstances estimates of $k$, the number of factors based on the early generations of a cross, are expected to be low. In these data, for example, each gene or effective factor detected in the $\mathrm{F}_{2}$ had become on average ten by the $\mathrm{F}_{6}$ with no indication that any limit to the increase had been reached.

There are further reasons why the high cstimates in the $F_{6}$ may still be an underestimate, namely, that the data were not collected from an optimally designed experiment for this purpose or from plants grown under optimal conditions. Thus plots not single plants were the units of randomisation and much of the data was collected during the initial stages of developing a new experimental site. Both reduced the sensitivity with which segregation could be detected and hence our estimates of $k$.

For the $\mathrm{F}_{2}$, but not for the other generations, we were able to compare three different ways of using genotype assay for estimating $k$. Two of these, namely those based upon $p=2$ and $p=4$, are strictly comparable in that each estimate is based on the same total number of plants. The third based on lineages, combines all the information from all the generations. The latter method, as might be expected, gave higher estimates than those obtained for $p=2$, and for FT but not FH the estimates for $p=4$ were also higher.

The present analyses confirm the overwhelming superiority of genotype assay over all other methods of estimating the number of effective factors that are available for all except a few species. The better of the previous estimates for FT and FH for the $\mathrm{Vl} \times \mathrm{V} 5$ cross have ranged between three and seven and the highest previous estimates based upon $\mathrm{F}_{10}$ inbreds were seven for FT and five for FH (Perkins and Jinks, 1973).

Acknowledgments.-We are grcatly indebted to Mr P. J. Jinks for generalising the probability formulac. One of us (P. T.) is supported by an S.R.C. Research Studentship.

\section{REFERENGES}

BREESE, E. L. 1954. Continuous variation in higher plants. Ph.D. Thesis, University of Birmingham.

BUCIO AI.ANIS, L., PERKINS, J. M., AND JINKS, J. L. 1969. Environmental and genotypeenvironmental components of variability. V. Segregating generations. Heredity, 24, 115-127.

JINKS, J. L., AND TOWFY, PHILOMENA. 1976. Fistimating the number of genes in a polygenic system by genotype assay. Heredity, 37, 69-81.

Matiler, K., AND JINKS, J. L. 1971. Biometrical Genetics. 2nd Fdition. Chapman and Hall, London.

Mather, K., AND vines, A. 1952. The inheritance of height and flowering time in a cross of Nicotiana rustica, from Quantitative Inheritance, 49-80. H.M.S.O., London.

PERKINS, J. M., AND JINKS, J. L. 1970. Detection and estimation of genotype-crivironmental, linkage and cpistatic components of variation for a metrical trait. Heredity, 25, 157177.

PERKINS, J. M., AND JINKs, J. L. 1973. The assessment and specificity of environmental and genotype-environmental components of variability. Heredity, 30, 111-126. 